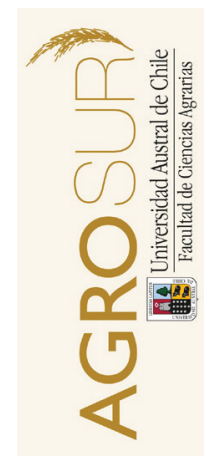

\title{
Influencia de la época de parto sobre la producción y calidad de leche de un predio con estabulación completa en la Región de los Ríos, Chile
}

\author{
Influence of calving season on milk production and composition in \\ a free stall farm in los Rios Region, Chile \\ Petres, C. $^{a}$, Mujica, $\mathrm{F}^{b *}$, Uribe, $\mathrm{H}^{c}{ }^{c}$ \\ ${ }^{a}$ curthler.petres@gmail.com \\ ${ }^{b}$ Instituto de Producción Animal, Universidad Austral de Chile. \\ ${ }^{c}$ Departamento Producción Animal, Universidad de Chile.
}

\begin{tabular}{l} 
A R T I C L E I N F O \\
\hline Keywords: \\
Holstein Friesian \\
Free stall \\
Calving season \\
Milk production \\
Fat production \\
Protein production \\
\hline
\end{tabular}

Original Research Article, Animal Science

${ }^{*}$ Corresponding author:

Curthler Petres

E-mail address:

fernandomujica@uach.cl

\begin{abstract}
A B S T R A C T
The study was conducted on a farm with Holstein Friesian cows kept in complete free stall-housing in the Cudico area of Los Ríos Region of Chile. The data were milk records from the Cooperativa de Inseminación Artificial (COOPRINSEM), which consisted of 955 lactations made from 2011 to 2014. The general objective was to determine the influence of calving season on yield. Additionally milk quality, somatic cell count and urea across. Data were analyzed using the SAS software by PROC MEANS and PROC GLM modules.

The results show that calving season had a significant influence $(\mathrm{p} \leq 0.01)$ on milk, fat and protein yield, being higher, for milk and fat yield, in cows calving on Fall, 11,932 and $430 \mathrm{~kg}$ respectively; while the protein was higher for Summer calving cows at $413 \mathrm{~kg}$. In more than half of the lactations (52.9\%), milk yield reached between 11,001 to 12,500 kg of milk. Yields were higher in 2012, 2011 and 2014 for milk, mil fat and milk protein, respectively.

On regard to milk hygienic quality, measured as somatic cell count (RCS), age and calving year were significant. However, age and calving year were not significant for urea.
\end{abstract}

\section{RESUMEN}

El estudio fue realizado con estabulación completa de la localidad de Cudico, Región de los Ríos, Chile. En base a registros de control lechero proporcionados por la Cooperativa de Inseminación Artificial (COOPRINSEM), el cual constaba de 955 lactancias entre el año 2011 al 2014. El objetivo general fue determinar la influencia de la época de parto sobre los rendimientos productivos. Se analizó adicionalmente la producción y calidad de leche, recuento de células somáticas y urea a través de los años.

Los resultados obtenidos reflejan que la época de parto tuvo influencia significativa $(\mathrm{p} \leq 0,01)$ en la producción de leche, grasa y proteína, siendo mayores durante el otoño para la producción de leche y grasa con 11.932 y 430 kg respectivamente; mientras que la proteína fue mayor durante el verano con $413 \mathrm{~kg}$. Se alcanzó una producción de leche entre 11.001-12.500 kg de leche, siendo mayor durante el año 2012, la producción de grasa fue mayor entre el 2011-2012, en tanto la proteína no presenta diferencias significativas en los años de estudio.

En relación a la calidad higiénica de la leche, medido como el recuento de células somáticas (RCS), la época y el año de parto, fueron significativas, no así para el caso de la urea.

Palabras clave: Raza Holstein Friesian, estabulación, época de parto, producción de leche, producción de grasa, producción de proteína.

\section{INTRODUCCIÓN}

En Chile la zona sur se destaca por su marcada producción lechera a nivel país, ya que comprende 6.000 productores comerciales de leche, especialmente concentrados en las regiones de Los Ríos y Los Lagos.

En el ámbito de la calidad de la leche, se debe tener en cuenta que ésta puede sufrir algún tipo de alteración durante el transcurso de la lactancia, ya sea por moti- vos de alimentación de la vaca, por influencia racial o factores externos.

Por lo anterior, la fecha de parto elegida por el productor es sujeta a cambios e influye en la producción de leche a alcanzar, considerándose esta como una herramienta de manejo en el plantel.

Este estudio se realizó usando datos de animales que se encuentran bajo un sistema de estabulación completa, pero con mayores costos al requerir mayor 
mano de obra para realizar tareas de limpieza y alimentación, además de un mayor capital inicial, comparado con un sistema tradicional de pastoreo. (Arronis, 2001).

La característica principal de esta raza es la capacidad que tiene de convertir con mayor eficiencia el alimento en leche, basándose su alimentación en recursos forrajeros de mayor nivel nutricional como granos y cultivos suplementarios de invierno y verano. En los últimos 5 años la raza en Chile se ha visto reforzada con nuevo aporte genético de líneas europeas de las cuales destacan líneas alemanas y holandesas. (SOFO, 2012).

El objetivo general de este estudio es determinar la influencia de la época de parto sobre los rendimientos productivos de vacas.

\section{MATERIAL Y MÉTODO}

Para llevar a cabo este estudio, se utilizaron registros del control lechero de un plantel de 559 vacas de la raza Holstein, corresponde a un registro mensual, facilitado en forma digital en una planilla Excel, por la Cooperativa de Inseminación Artificial (COOPRINSEM). De un total de 911 lactancias de la base de datos, quedaron 811 disponibles para analizar, tras ejecutar los criterios de eliminación correspondientes. (Lapso interparto > 600 días y días de lactancia $<100$ días).

En cuanto al manejo alimenticio, la dieta está conformada principalmente por soiling de ballica $(36,5 \mathrm{~kg})$, silo de maíz (17,3 kg con 28\% MS), maíz grano húmedo, soja, maíz roleado, silo de alfalfa, heno de alfalfa, maíz molido fino y minerales, todos estos escritos en forma decreciente.

El análisis de los datos se realizó primero por medio de estadística descriptiva usando el módulo PROC MEANS del programa Statistical Analysis System (SAS) y luego usando regresión lineal múltiple mediante el módulo PROC GLM de SAS (SAS Copyright (C), 20022003).

En el predio, los partos ocurren durante todo el año. Para facilitar el manejo de los datos, las vacas se agruparon en categorías de: época de parto, recuento de células somáticas, lapso inter parto y grupo de urea, asignándole una sigla a cada variable y un nivel (Cuadro 1).

Los datos se analizaron con modelos estadísticos para cuantificar la influencia de determinadas variables, especialmente épocas de parto, sobre producción de leche, grasa y proteína. Además se probaron interacciones entre variables que serán explicadas posteriormente. Las variables disponibles en la base de datos, corresponden a:

Época de parto (EPOCA)

Producción de leche (LECHE)

Recuento células somáticas (RCS)

Producción de urea (UREA)
Producción de grasa (GRASA)

Número ordinal de parto (NOP)

Producción de proteína (PROTEINA)

Año de parto (APARTO)

Lapso inter parto (CLIP)

Días de lactancia (DL)

\section{Modelos estadísticos}

Los variables dependientes que fueron analizadas mediante modelos lineales son producción de leche, producción de grasa, producción de proteína, RCS y urea. Aquellas variables que no resultaron significativas $(p \geq 0,05)$ fueron removidas del modelo.

\section{Modelo producción de leche:}

$\mathrm{Y}_{i j k l m n o}: \mu+\mathrm{EPOCA}_{\mathrm{i}+} \mathrm{APARTO}_{\mathrm{j}}+\mathrm{RCS}_{\mathrm{k}}+\mathrm{CLIP}_{1}+\mathrm{NOP}_{\mathrm{m}}+$ $\mathrm{UREA}_{\mathrm{n}}+\mathrm{bDL}_{\mathrm{jklmno}}+\mathrm{e}_{\mathrm{jklmnop}}$

\section{Donde:}

$\mathrm{m}=\mu=$ intercepto general.

$\mathrm{b}=$ coeficiente de regresión de producción de leche en días de lactancia.

$\mathrm{DL}_{\mathrm{jklmno}}=D L_{\text {jklmnop }}=$ son los días de lactancia de la ijklmno-ésima observación.

\section{Modelo producción de grasa:}

$\mathrm{Y}_{i j k l m n}: \mu+$ EPOCA $_{\mathrm{i}}+\mathrm{APARTO}_{\mathrm{j}}+\mathrm{CLIP}_{\mathrm{k}}+\mathrm{NOP}_{1}+$ $(\text { APARTO*NOP) })_{j 1}+b L_{j k l m}+\mathrm{e}_{i j k l m n}$

\section{Modelo producción de proteína:}

$\mathrm{Y}_{i j k l m n o}: \mu+\mathrm{EPOCA}_{\mathrm{i}} \mathrm{APARTO}_{\mathrm{j}}+\mathrm{RCS}_{\mathrm{k}}+\mathrm{CLIP}_{\mathrm{l}}+\mathrm{NOP}_{\mathrm{m}}+$ $\mathrm{bDL}_{\mathrm{jklmn}}$

\section{Modelo RCS:}

$\mathrm{Y}_{\text {ijklmnop }}: \mu+\mathrm{EPOCA}_{\mathrm{i}}+\mathrm{APARTO}_{\mathrm{j}}+\mathrm{CLIP}_{\mathrm{k}}+\mathrm{NOP}_{1}+\mathrm{LECHE}_{\mathrm{m}}$ + GRASA $_{\mathrm{n}}+$ PROTEINA $_{\mathrm{o}}+\mathrm{e}_{\mathrm{ijklmnop}}$

Donde:

LECHE $_{\mathrm{m}}$, GRASA $_{\mathrm{n}}$ y PROTEINA $=$ utilizadas como covariable.

\section{Modelo producción de urea:}

$\mathrm{Y}_{\text {iiklmnopg }}: \mu+\mathrm{EPOCA}_{\mathrm{i}}+\mathrm{APARTO}_{\mathrm{j}}+\mathrm{CLIP}_{\mathrm{k}}+\mathrm{NOP}_{1}+\mathrm{RCS}_{\mathrm{m}}+$ LECHE $_{\mathrm{n}}+$ GRASA $_{\mathrm{o}}+$ PROTEINA $_{\mathrm{p}}+\mathrm{e}_{\mathrm{ijklmnopq}}$

Donde:

LECHE $_{\mathrm{m}}$, GRASA $_{\mathrm{n}}$ y PROTEINA $=$ utilizadas como covariable.

\section{RESULTADOS Y DISCUSIÓN}

En el Cuadro 2, se presentan los promedios de las variables utilizadas en relación a la época de parto a partir del análisis descriptivo, indicando la media y desviación estándar obtenidos para cada una. 
Cuadro 1. Agrupaciones y niveles conformados según la época de parto RCS, LIP y GUREA.

Table 1. Groupings and levels conformed according to the time of delivery RCS, LIP and GUREA.

\begin{tabular}{|c|c|c|c|c|}
\hline Variable & Sigla & Agrupación & Época & Nivel \\
\hline Época de parto & Época & Diciembre - Febrero & Verano & 1 \\
\hline Época de parto & Época & Marzo - Mayo & Otoño & 2 \\
\hline Época de parto & Época & Junio - Agosto & Invierno & 3 \\
\hline Época de parto & Época & Septiembre - Noviembre & Primavera & 4 \\
\hline Recuento células somáticas & RCS & $0-100 \mathrm{cel} / \mathrm{ml}$ & RCS1 & 1 \\
\hline Recuento células somáticas & RCS & $101-200 \mathrm{cel} / \mathrm{ml}$ & RCS2 & 2 \\
\hline Recuento células somáticas & RCS & $201-1500 \mathrm{cel} / \mathrm{ml}$ & RCS3 & 3 \\
\hline Categoría lapso interparto & LIP & 0-380 días & LIP1 & 1 \\
\hline Categoría lapso interparto & LIP & 381- 440 días & LIP2 & 2 \\
\hline Categoría lapso interparto & LIP & 441- 600 días & LIP3 & 3 \\
\hline Grupo urea & GUREA & $0-320 \mathrm{mg} / \mathrm{kg}$ & GUREA1 & 1 \\
\hline Grupo urea & GUREA & $321-400 \mathrm{mg} / \mathrm{kg}$ & GUREA2 & 2 \\
\hline Grupo urea & GUREA & $>401 \mathrm{mg} / \mathrm{kg}$ & GUREA3 & 3 \\
\hline
\end{tabular}

Cuadro 2. Promedio, desviación estándar, valores máximos y mínimos y número de observaciones de producción de leche, grasa y proteína a través de época de parto.

Table 2. Average, standard deviation, maximum and minimum values and number of observations of milk, fat and protein production through the time of calving.

\begin{tabular}{|c|c|c|c|c|c|}
\hline Variable & $\mathbf{N}$ & Media & Desv. Estándar & Mínimo & Máximo \\
\hline${ }^{1}$ Prod. Leche (kg) & 811 & & & & \\
\hline Verano & 63 & 11.742 & 3.556 & 3.194 & 20.802 \\
\hline Otoño & 241 & 11.932 & 4.153 & 1.329 & 28.347 \\
\hline Invierno & 224 & 11.199 & 3.797 & 2.039 & 21.643 \\
\hline Primavera & 283 & 11.343 & 3.428 & 1.463 & 21.292 \\
\hline Prod. Grasa (kg) & 811 & & & & \\
\hline Verano & 63 & 416,9 & 154,8 & 40,1 & 740,2 \\
\hline Otoño & 241 & 430,4 & 151,7 & 62,9 & 968,5 \\
\hline Invierno & 224 & 411,9 & 161,0 & 83,5 & 860,0 \\
\hline Primavera & 283 & 381,2 & 149,5 & 62,4 & 830,9 \\
\hline Prod. Proteína (kg) & 811 & & & & \\
\hline Verano & 63 & 413,7 & 119,5 & 115,4 & 707,7 \\
\hline Otoño & 241 & 397,0 & 139,3 & 32,7 & 829,6 \\
\hline Invierno & 224 & 382,5 & 131,7 & 67,7 & 745,5 \\
\hline Primavera & 283 & 383,3 & 116,3 & 39,5 & 762,5 \\
\hline
\end{tabular}

${ }^{1}$ Produccion láctea total, sin estandarización a 305 días. 


\section{Efecto de la época de parto sobre la producción de leche}

En el Cuadro 3 se presentan los promedios mínimos cuadrados de producción de leche para cada época de parto, se observa que la época de parto tuvo un efecto significativo en la producción de leche. Siendo las épocas de otoño-invierno las que alcanzan una mayor producción, siendo esto consecuencia de la alimentación. Esto se explica porque es en otoño donde se realiza la cosecha de maíz, siendo un ingrediente importante en la ración, por lo que el maíz húmedo que se entrega permite obtener mayores rendimientos productivos en dichas épocas al tener un mayor contenido nutritivo en fresco. Se registran menores producciones en lactancias iniciadas durante la primavera, ya que en la dieta el silo de maíz se da en menor cantidad y contiene un mayor porcentaje de MS, lo cual provoca una disminución de nutrientes para el animal, esto se contradice a los sistemas tradicionales de pastoreo que obtienen mayor producción en esta época, además presenta diferencias significativas en relación con otoño e invierno, que alcanzan mayores producciones.

\section{Efecto de la época de parto sobre la producción de grasa}

La producción de grasa, fue mayor en el otoño y el invierno, obteniendo una media de $432 \mathrm{~kg}$ y $427 \mathrm{~kg}$ e inferior durante la primavera y verano, con $373 \mathrm{~kg}$ y $391 \mathrm{~kg}$, respectivamente (Cuadro 3).

Morales (1999) indica que, en lecherías con estabulación completa, hay variaciones estacionales en la composición de la leche, pero este efecto está originado por la etapa de la lactancia de las vacas. Aún así, se reconoce que hay un efecto de la estación del año sobre el porcentaje de grasa de la leche, presentando los meses de verano $0,4 \%$ menos grasa que los meses de invierno, lo que confirma los resultados obtenidos en este estudio.

Cuadro 3. Promedios mínimos cuadrados (PMC), grasa y proteína según la época de parto.

Table 3. Square minimum averages (PMC), fat and protein according to the time of calving.

\begin{tabular}{cccc}
\hline Grupos & PMC Leche & PMC Grasa & PMC Proteína \\
\hline Verano & $11.193,99^{\mathrm{ab}}$ & $391^{\mathrm{b}}$ & $388^{\mathrm{ab}}$ \\
Otoño & $11.791,35^{\mathrm{a}}$ & $432^{\mathrm{a}}$ & $398^{\mathrm{a}}$ \\
Invierno & $11.651,06^{\mathrm{a}}$ & $427^{\mathrm{a}}$ & $397^{\mathrm{a}}$ \\
Primavera & $11.108,00^{\mathrm{b}}$ & $373^{\mathrm{b}}$ & $375^{\mathrm{b}}$ \\
\hline
\end{tabular}

Letras distintas indican diferencias significativas $(\mathrm{p}<0,05)$.

\section{Efecto de la época de parto sobre la producción de proteína}

La producción de proteína presenta una media mínima cuadrática que es máxima en otoño con $398 \mathrm{~kg}$ y un valor inferior en primavera con $375 \mathrm{~kg}$. Además no hubo diferencias entre los partos ocurridos en verano, otoño ni invierno (Cuadro 3).

Los resultados obtenidos en el presente estudio no coinciden con Anrique y Bidegain (2012), donde en lecherías con parto estacional; señala que normalmente durante la época de primavera se produce un aumento en la producción de proteína, lo cual se debe a una mejor calidad del forraje.

\section{Análisis de las variables productivas a través de los años de estudio}

En el Cuadro 4 se presentan los resultados del análisis descriptivo de las variables en estudios través de los años de estudio.

\section{Producción de leche}

La media y desviación estándar para el año con mayor producción (año 2012), coincide con el rendimiento de la Región de los Ríos durante ese año. Según ODEPA (2012) a nivel regional el crecimiento en la recepción fue liderado por la región de Los Ríos teniendo un alza en la producción de 50 millones de litros.

En el Cuadro 5, según los PMC para producción de leche, no se obtuvo diferencias significativas entre los años 2011- 2012- 2013, pero sí en el año 2014, que fue donde se presentaron las menores producciones.

\section{Producción de grasa}

La producción de grasa fue significativamente mayor durante los años 2011 y 2012, con 427 y $420 \mathrm{~kg}$ respectivamente e inferior para los años 2013 y 2014 con 382 y $380 \mathrm{~kg}$ respectivamente (Cuadro 5).

Al considerar los promedios mínimos cuadrados de producción de grasa para cada año de lactancia, en el Cuadro 5, se puede apreciar que entre los años 20112012 no hay diferencias significativas, lo mismo ocurre entre los años 2013-2014, pero sí se presentan diferencias significativas entre ambos grupos, lo cual se debe a las condiciones de producción en los respectivos años. Lo anterior explicado principalmente por la disminución de concentrado en la dieta en los últimos años, siendo en promedio de 9 kilos, disminuyendo a 5 kilos en la ración.

La interacción (APARTO*NOP), presentó significancia estadística para con la producción de grasa con el transcurso de los años. $(\mathrm{p}<0,01)$. 


\section{Producción de proteína}

La media y desviación estándar para el año con mayor producción (el año 2014) fue de 395,2 $\pm 93 \mathrm{~kg}$ (Cuadro 4).

La época de parto fue la única variable significativa para la producción de proteína; por lo que esta no presenta diferencias significativas a través de los años de lactancia comprendidos en este estudio (Cuadro 5).

Además la producción de proteína no presenta diferencias significativas según el número ordinal de parto y lapso interparto en este estudio ( $p>0,05)$.

Anrique y Bidegain (2012); señala que la calidad del forraje, el consumo de materia seca y de concentrado, son influyentes en los resultados a obtener.

Cuadro 4. Promedio, desviación estándar, valores máximos y mínimos y número de observaciones de producción de leche, grasa y proteína a través de años de producción.

Table 4. Average, standard deviation, maximum and minimum values and number of observations of milk, fat and protein production through years of production.

\begin{tabular}{lccccc}
\hline \multicolumn{1}{c}{ Variable } & N & Media & Desv. estándar & Mínimo & Máximo \\
\hline 'Prod. Leche año (kg) & $\mathbf{8 1 1}$ & & & & \\
Año 2011 & 201 & 11.209 & 4.466 & 1.329 & 23.415 \\
Año 2012 & 294 & 11.590 & 3.979 & 1.463 & 28.347 \\
Año 2013 & 229 & 11.527 & 3.090 & 3.649 & 21.292 \\
Año 2014 & 87 & 11.492 & 2.814 & 3.194 & 18.568 \\
Prod. Grasa (kg) & $\mathbf{8 1 1}$ & & & & \\
Año 2011 & 201 & 417,3 & 176,50 & 62,90 & 860,0 \\
Año 2012 & 294 & 416,0 & 159,91 & 62,40 & 968,5 \\
Año 2013 & 229 & 389,3 & 140,17 & 40,10 & 792,5 \\
Año 2014 & 87 & 400,1 & 113,80 & 93,00 & 711,3 \\
Prod. Proteína (kg) & $\mathbf{8 1 1}$ & & & & \\
Año 2011 & 201 & 380,5 & 155,12 & 45,10 & 829,6 \\
Año 2012 & 294 & 390,8 & 132,81 & 32,70 & 754,7 \\
Año 2013 & 229 & 393,6 & 106,04 & 132,50 & 762,5 \\
Año 2014 & 87 & 395,2 & 93,07 & 93,07 & 647,2 \\
Prod. RCS (cel/mL) & $\mathbf{8 1 1}$ & & & & \\
Año 2011 & 201 & 233,2 & 428,6 & 16 & 5.765 \\
Año 2012 & 294 & 172,1 & 163,6 & 18 & 1.500 \\
Año 2013 & 229 & 145,3 & 107,9 & 13 & 647 \\
Año 2014 & 87 & 157,0 & 163,7 & 28 & 1.360 \\
\hline
\end{tabular}

${ }^{1}$ Produccion láctea total, sin estandarización a 305 días.

Cuadro 5. Promedios mínimos cuadrados (PMC) para producción de leche, grasa, proteína, RCS y urea según los años de estudio. Table 5. Minimum least squares (PMC) for the production of milk, fat, protein, RCS and urea according to the study years.

\begin{tabular}{cccccc}
\hline Grupos & $\begin{array}{c}\text { PMC } \\
\text { Leche }\end{array}$ & $\begin{array}{c}\text { PMC } \\
\text { Grasa }\end{array}$ & $\begin{array}{c}\text { PMC } \\
\text { Proteína }\end{array}$ & $\begin{array}{c}\text { PMC } \\
\text { RCS }\end{array}$ & $\begin{array}{c}\text { PMC } \\
\text { UREA }\end{array}$ \\
\hline 2011 & $11.498^{\mathrm{a}}$ & $427^{\mathrm{a}}$ & $390^{\mathrm{a}}$ & $233^{\mathrm{a}}$ & $371^{\mathrm{a}}$ \\
2012 & $11.716^{\mathrm{a}}$ & $420^{\mathrm{a}}$ & $395^{\mathrm{a}}$ & $172^{\mathrm{b}}$ & $369^{\mathrm{a}}$ \\
2013 & $11.328^{\mathrm{a}}$ & $382^{\mathrm{b}}$ & $386^{\mathrm{a}}$ & $145^{\mathrm{b}}$ & $362^{\mathrm{a}}$ \\
2014 & $10.920^{\mathrm{b}}$ & $380^{\mathrm{b}}$ & $376^{\mathrm{a}}$ & $157^{\mathrm{b}}$ & $363^{\mathrm{a}}$ \\
\hline
\end{tabular}

Letras distintas indican diferencias significativas $(\mathrm{p}<0,05)$. 


\section{Recuento de células somáticas (RCS)}

El RCS fue significativamente mayor, los siguientes tres años no presentó diferencias significativas (Cuadro 5).

El RCS fue afectado significativamente solo con la época y el año de parto $(p<0,01)$.

En relación a la época de parto, fue mayor el RCS durante el invierno, lo que se explica mayormente por el manejo del rebaño. En esta época la posibilidad de contaminación entre animales, la menor higiene que hay en la sala de ordeña y en los establos, a pesar de que se realice aseo periódicamente, la pluviometría incide en la formación de barro lo que promueve el RCS en la leche y la cantidad de bacterias (Estafilococos aureus, Estreptococos agalactiae) presentes en el ambiente.

Pedraza et al. (2000), indican que entre la producción de leche y RCS, existe una relación inversa, es decir que a mayor cantidad de RCS es menor la producción de leche o viceversa. No se cumple en este estudio, ya que la producción de leche no se ve influenciada por un mayor RCS.

Lo anterior se explica tras el resultado del modelo de RCS, al presentar la leche $p>0,05$ demostrando no ser significativa, explicado por los bajos niveles de RCS, en la lechería analizada (Cuadro 5).

\section{Producción de Urea}

A lo largo de los años, no se evidenciaron diferencias significativas. Estando dentro del rango ideal de urea que va entre $150-380 \mathrm{mg} / \mathrm{kg}$.

González y Vázquez (2000), señalan que la urea es un producto final del metabolismo de las proteínas. La proteína que la vaca no utiliza para su mantenimiento y producción, se descompone en amoníaco, que es muy tóxico para las células, convirtiéndose en urea en el hígado que entra en el flujo sanguíneo. Cuando se produce urea, esta se difunde en todos tejidos del cuerpo de la vaca y aparece en la leche. El ideal de concentración de urea en leche coincide con los resultados del presente estudio.

Se puede apreciar que el nivel de urea presente en la leche de éste plantel es óptimo, ya que no supera los $380 \mathrm{mg} / \mathrm{kg}$, indicando que la dieta de las vacas tiene una óptima proporción de proteína. Las diferencias de producción de urea en leche no fueron estadísticamente significativas entre los años de estudio. (Cuadro 5).

\section{CONCLUSIONES}

En base a los resultados obtenidos del estudio realizado se concluyó que la época de parto tiene influencia sobre la producción de leche, grasa y proteína, por lo que es una herramienta de manejo a tener en cuenta, ya que la producción de cualquiera de estos tres componentes se relaciona con la rentabilidad que genera el rebaño lechero.

La producción de leche se mantiene en niveles óptimos durante los tres primeros años de estudio, disminuyendo en el último año evaluado, bajo el promedio ideal de la raza utilizada en este estudio.

La evolución de la composición de leche, es decir grasa y proteína, presenta disminución con el paso de los años, lo cual se explica por un menor aporte de concentrado en la dieta, lo que provoca una baja en la entrega de nutrientes y energía para los animales.

La calidad higiénica de la leche, en base al recuento de células somáticas, presenta un progreso a partir del segundo año de estudio, lo cual se debe a mejoras en el manejo del rebaño. Asimismo no presenta influencia en los rendimientos productivos como sucede comúnmente en animales a pastoreo.

La producción de urea, tiene niveles persistentes con el paso de los años, pudiendo ser explicado por la dieta equilibrada y poco variable durante el año.

\section{REFERENCIAS}

Anrique, R., Bidegain, H., 2012. Inventario de capacidades de procesamiento de la industria lechera nacional actualización 2012. http://www.consorciolechero.cl/chile/ documentos/publicaciones/inventario-industria-lechera.pdf

Arronis, V., 2001. Recomendaciones sobre sistemas intensivos de producción de carne: estabulación, semiestabulación y suplementación estratégica en pastoreo. Ministerio de Agricultura y Ganadería, Costa Rica, pp. 1-24. http://www.mag.go.cr/biblioteca_virtual_animal/estabulacion.pdf

González, A., Vázquez, O., 2000. El análisis de urea en leche como indicador del balance nutritiva de la alimentación de las vacas. Centro Investigacions Agrarias Mabegondo, Instituto Lácteo Gandeiro Galego, pp. 1-14. http://www. laboratoriollamas.com.ar/articulos/bovinos/Urea\%20 en $\% 20$ Leche $\% 20$ como\%20indicador $\% 20$ de $\% 20$ nutricion.pdf

Morales, M.S., 1999. Factores que afectan la composición de la leche. http://www.tecnovet.uchile.cl/index.php/RT/ article/view/5224/5104 (acceso, 05.2015).

Oficina de Estudios y Políticas Agrarias (ODEPA), 2012. Leche y derivados. http://www.odepa.cl/rubro/leche-yderivados/

Pedraza, C., Mansilla, A., Fajardo, P., Agüero, H., 2000. Cambios en la producción y composición láctea por efecto del incremento de células somáticas en leche de vacas. Agricultura Técnica 60, 251-258.

Sociedad de Fomento Agrícola de Temuco A.G. (SOFO), 2012. Razas, Holstein Friesian (HF). http://www.sofo.cl/ _web/_servicios/_registro/r_bovinos/holstein.htm 